\title{
Automatic Burst Detection based on Line Length in the Premature EEG
}

\author{
Ninah Koolen ${ }^{1,2}$, Katrien Jansen ${ }^{3}$, Jan Vervisch ${ }^{3}$, Vladimir Matic ${ }^{1,2}$, Maarten De Vos ${ }^{1,2,4}$, \\ Gunnar Naulaers ${ }^{5}$ and Sabine Van Huffel ${ }^{1,2}$ \\ ${ }^{I}$ Department of Electrical Engineering (ESAT), division SCD, Katholieke Universiteit Leuven, Leuven, Belgium \\ ${ }^{2}$ iMinds-KU Leuven Future Health Department, Leuven, Belgium \\ ${ }^{3}$ Department of Pediatrics, University Hospital Gasthuisberg, Leuven, Belgium \\ ${ }^{4}$ Department of Psychology, University of Oldenburg, Oldenburg, Germany \\ ${ }^{5}$ Neonatal Intensive Care Unit, University Hospital Gasthuisberg, Leuven, Belgium \\ \{ninah.koolen, vladimir.matic, maarten.devos, sabine.vanhuffel\}@esat.kuleuven.be, \\ \{katrien.jansen, jan.vervisch, gunnar.naulaers\}@uzleuven.be
}

Keywords: Brain Monitoring, Premature EEG, Automatic Detection, Burst, Interburst Interval, Neonatal Intensive Care Unit.

\begin{abstract}
To extract useful information from preterm electroencephalogram (EEG) for diagnosis and long-term prognosis, automated processing of EEG is a crucial step to reduce the workload of neurologists. Important information is contained in the bursts, the interburst-intervals (IBIs) and the evolution of their duration over time. Therefore, an algorithm to automatically detect bursts and IBIs would be of significant value in the Neonatal Intensive Care Unit (NICU). The developed algorithm is based on calculation of the line length to segment EEG into bursts and IBIs. Validating burst detection of this algorithm with expert labelling and existing methods shows the robustness of this algorithm for the patients under test. Moreover, automation is within our grasp as calculated features mimic values obtained by scoring of experts. The outline for successful computer-aided detection of bursting processes is shown, thereby paving the way for improvement of the overall assessment in the NICU.
\end{abstract}

\section{INTRODUCTION}

Premature infants are at high risk for neurological disorders. Electroencephalography (EEG) indicates both the nature and the location of the pathogenesis. It would be very helpful, in addition to the visual inspection of time-consuming EEG by neonatologists, to develop an automatic algorithm that quantifies the brain activities and its evolution.

Despite the fact that EEG is already widely used for registration of brain processes for epilepsy patients, (semi-)automated monitoring of quantitative EEG variables and its validated use is almost nonexistent. Moreover, there is a high need for automatic analysis of the neonatal EEG to significantly reduce the workload of clinicians in the NICU. In this paper, an algorithm is developed for premature infants. For these patients it is very important to monitor EEG within the first six hours after birth to make an accurate prognosis on survival quality and neurological outcome.
This diagnosis is based on the 'hidden' information in the so-called background EEG activity. Critical factors for prognosis are amplitude and the degree of (dis-)continuities of the background EEG (Vanhatalo and Kaila, 2006).

Furthermore, specifically abnormal patterns can be observed. Discontinuous EEG pattern, or the socalled trace discontinue, consists of bursts with high frequencies and high amplitude, interrupted by periods of low brain activity with low-voltage EEG, named the interburst intervals (IBIs). It is believed that long low-voltage periods give rise to an increased risk of brain dysfunctions (Le Bihannic et al., 2011). Nevertheless, good neurological outcome can be expected if low-voltage activity recovers into increasing activity between bursts and evolve to a normal pattern within 12 hours after birth. However, there is no golden standard for the description of bursts in the literature, so validation of the detection algorithm is subjective. In this way, validation should be performed by more than one clinician and 
the experience of experts should be combined.

Earlier, automatic detection of bursts was often only based on a threshold on the amplitude of the EEG channels, but this has some drawbacks. High frequency artefacts which are not filtered out can be seen as bursts, whereas medication and filter settings can also influence the amplitude of the EEG. Recent studies include besides amplitude content also frequency content of bursts and IBIs, e.g. they make use of a non-linear energy operator (Särkelä et al., 2002); (Palmu et al., 2010) or threshold detection on the envelope of the EEG channels (Jennekens et al., 2011).

The goal of this study was to implement a reliable detection algorithm with the focus on the advantage of combining amplitude and frequency content. Therefore, line length is introduced for this application, because it is very accurate in detecting the onset of high activity in the EEG (Esteller et al., 2001). Moreover, the developed method allows defining an adaptive and patient specific threshold. This avoids the limitations of changing amplitudes' level, e.g. when medication is administrated. Furthermore, no training set is needed. Such an algorithm in the NICU would allow a more objective analysis. Future research will also investigate the influence of additional artefact removal as proposed in (De Vos et al., 2011) on the accuracy of burst and IBI detection. The computer-aided analysis of the EEG enables to reduce the cost for the timeconsuming long-term analysis, and thereby reduces the risk of brain damage of preterm infants.

\section{DATA ACQUISITION}

The EEG was measured with OSG equipment at 9 electrode locations (Fp1, Fp2, T3, T4, C3, C4, Cz, $\mathrm{O} 1, \mathrm{O} 2)$ and sampling frequency of $250 \mathrm{~Hz}$. The polysomnographic dataset included long-term videoEEG recordings of 5 preterm infants with a postmenstrual age of 24-32 weeks. Two patients had measurements at 3 different moments in time to see an evolution in the brain development. These moments are as soon as possible after birth, at day 14 and at the day when the patient could leave the hospital. The protocol was approved by the ethics committee of the University Hospitals of Leuven, Belgium. First, a pre-processing step is performed; a 50 and 100 Notch filter and a 1-20 Hz band pass filter are applied. After this step, twenty minutes of each EEG were chosen for further analysis and scored (burst/IBI) by two experienced clinicians.

\section{METHODOLOGY}

\subsection{Detection based on Line Length}

Fractal dimension (FD) is a promising method for transient detection, requiring no prior knowledge of the characteristics of the transient (Accardo et al., 1996). As the dimension of a line is 1 and for a plane 2 , the FD in EEG will always be between 1 and 2 . The more the line fluctuates, the more the plane is 'covered', so the more the FD increases. The line length is a simplified version of the FD. Line length is more successful than FD for burst detection. It is also reported (Esteller et al., 2001) for the detection of seizures in the EEG. The line length is the running sum of the absolute differences between all consecutive samples within a predefined window. An efficient burst detection algorithm is derived from this feature. The algorithm consists of the following steps:

1. Segmentation of each EEG channel in consecutive segments of 1 second, with an overlap of 0.12 second (Figure 1) (Accardo et al., 1996). To have reliable detection of transient events like bursts, short duration segments are necessary. Line length will grow as the data sequence magnitude or signal variance increases. Hence, it can be seen as an amplitude and frequency demodulator (Esteller et al., 2001).

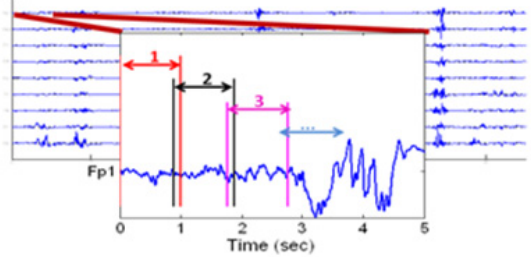

Figure 1: Partition of the EEG signals in overlapping consecutive segments of 1 second. The overlap is 0.12 second.

2. For each segment $i$ of each channel $n$, the total length is calculated as in formula 1. L(i) represents the line length value for each segment $i$, calculated as the sum of the distances between successive data samples $x_{j}$ within this segment.

$$
\mathrm{L}(\mathrm{i})=\sum_{\mathrm{j}=1}^{250-1}\left|\mathrm{x}_{\mathrm{j}+1}-\mathrm{x}_{\mathrm{j}}\right|
$$

After that, these line lengths are normalized by the total sum of the line lengths of that EEG channel $n$ (Accardo et al., 1996):

$$
\mathrm{L}_{\mathrm{n}}(\mathrm{i})=\mathrm{L}(\mathrm{i}) / \sum_{\mathrm{i}} \mathrm{L}(\mathrm{i})
$$


Finally, the median value over all channels is taken for each segment (median $L_{n}(i)$ ), which is the (blue) curve in Figure 2 part c. Median value is more robust than the mean value because high amplitude or frequency content in only one channel would influence the mean value too much. Thereby, experts define bursts as high activity on more than one channel (or more than half of the EEG channels).

3. Bursts are detected when the amplitude of this curve (median $L_{n}(i)$ ) is above Thr_Det. This patient dependent threshold is calculated as $0.85^{*}$ mean of this curve. An additional condition for detection is that the difference in amplitude between a successive non-detected and detected point (and vice versa) should be large enough $\left(>0.4 * \operatorname{std}\left(\right.\right.$ median $\left.L_{n}(i)\right)$, so only pronounced peaks are detected. Additionally, all IBIs shorter than 2 seconds are removed as they are also not considered by clinical experts. For an example of 65 seconds EEG, this leads to detection of the segments indicated by red circles in Figure $2 \mathrm{c}$. In part $b$ of Figure 2, the detected bursts are compared with clinical labelling of high activity.

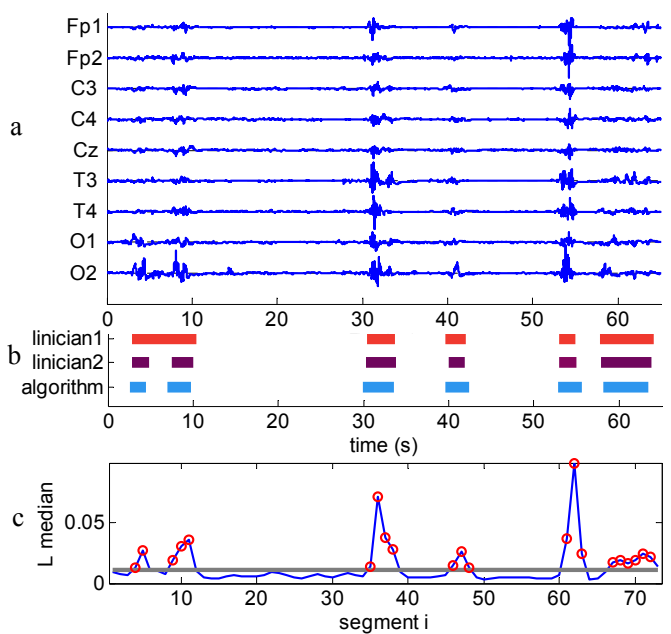

Figure 2: a) Example of 65 seconds 9-channel EEG recording, b) Burst detection: by 2 clinicians and by algorithm, c) Blue curve: median $L_{n}(i)$ as calculated in step 2, grey line: threshold for detection of bursts $(\mathrm{Thr}$ Det $)$, red circles: detected bursts after step 3 .

\subsection{Comparison of Detection Methods}

Jennekens et al.(2011) first calculate the envelope values $E V(i)$, which are derived from the average signal power $P(i)$ as in formula $3 . x(i)$ is the amplitude of the signal and $N w$ a window length equal to the number of sample points in 1 second of data. When these envelope values are obtained for every EEG channel, an amplitude-threshold is applied. If point $E v(i)$ has a value higher than this threshold on two or more channels, this sample $x(i)$ is detected as a burst sample. IBIs shorter than two seconds are removed.

$$
\mathrm{EV}(\mathrm{i})=\sqrt{2 \mathrm{P}(\mathrm{i})}=\sqrt{\frac{2}{\mathrm{Nw}} \sum_{\mathrm{i}=1}^{\mathrm{NW}} \mathrm{x}(\mathrm{i})^{2}}
$$

Another non-linear method is explored. It makes use of the non-linear energy operator (formula 4) (Palmu, 2010), where $i$ is the current sample and $x(i)$ the value at that sample.

$$
\operatorname{NLEO}(x(i))=|x(i) x(i-3)-x(i-1) x(i-2)|
$$

Thereafter, the processed signal is smoothed by the average value of a sliding window of 1.5 second centred at the time sample $N L E O(x(i))$. To remove continuous artefacts, a baseline correction is done by subtracting the minimum value of the smoothed signal from 1 minute epoch before the current sample. As in the previous method, marking as a burst is performed when the sample has a value higher than a predefined amplitude on two or more channels.

We compared those two methods with the developed burst detection algorithm, by comparing the accuracy of detection (to clinical labelling) and different features describing these epochs. The accuracy is calculated sample by sample, where a true positive (TP) is found as a sample $x(i)$ which is both by the algorithm and by the clinician detected as a burst. A true negative stands for a sample $x(i)$ which is marked as an interburst interval by the expert and by the algorithm. Then, the accuracy is calculated as in formula 5 .

$$
\text { accuracy }=(\mathrm{TP}+\mathrm{TN}) / \# \text { samples }
$$

\subsection{Features Describing Epochs}

To see an evolution of the EEG pattern of the premature brain, we will look at parameters which describe bursts and IBIs. Namely, more bursts indicate more activity and more connectivity between neurons in the premature brain.

In this paper, several parameters were used to compare epochs detected by different algorithms with clinical detected epochs (Palmu et al., 2010):

- Number of Bursts / IBIs: number of these specific epochs. It cannot be confused with the number of points in the EEG time series classified as bursts.

- Mean Burst / IBI Duration: average length of the burst or interburst interval. 
- Median Burst / IBI Duration: middle value of a finite ordered list of these bursts or IBIs.

Burst\%: proportion of time covered by bursts.

Although these parameters are clinically relevant, they do not fully summarize the patient's state. Therefore, some additional parameters are explored (Särkelä et al., 2002) related to the energy within the burst / IBI:

- Average Bursts / IBIs Amplitude: average absolute amplitude values of the original EEG samples which are detected as a burst or an IBI. It is calculated for the bursts as in formula 6 :

$$
\frac{\sum_{\mathrm{n}=1}^{\text {\#channels }} \frac{\sum_{\mathrm{j}=1}^{\text {\#bursts }} \frac{\sum_{\mathrm{i}=1}^{\text {burst length }}|\mathrm{x}(\mathrm{i})|}{\text { burst length }}}{\text { \#bursts }}}{\text { \#channels }}
$$

Average Energy Operator Bursts / IBIs: averaged NLEO values characterize the burst suppression pattern. Formula 7 presents how to calculate this value for burst epochs.

$\frac{\sum_{\mathrm{n}=1} \frac{\sum_{\mathrm{j}=1}^{\# \text { chansts }} \frac{\sum_{\mathrm{i}=4}^{\text {burst length }}|\mathrm{x}(\mathrm{i}) \mathrm{x}(\mathrm{i}-3)-\mathrm{x}(\mathrm{i}-1) \mathrm{x}(\mathrm{i}-2)|}{\text { burst length }}}{\text { \#bursts }}}{\text { \#channels }}$

\section{RESULTS AND DISCUSSION}

\subsection{Accuracy of Detection Methods}

Validation of the different algorithms is performed by comparison of automatic versus manually indicated bursts. In Figure 3, we present accuracy for the three algorithms: 1 . based on line length, 2 . based on envelope calculation and 3 . based on NLEO.

It can be seen that the developed algorithm (meth1) performs similarly to the inter-rater agreement in almost all patients. The mean accuracy is respectively $83.8 \%$ for validation of meth 1 and $86.5 \%$ for inter-rater agreement. For patient 3 the inter-rater agreement is very high $(90.6 \%)$ and differs $5 \%$ from the automatic detection. In many cases, NLEO-based algorithm $(80.9 \%$ mean accuracy) performs similarly to the method based on line length calculation, but with the difference that the first method has a computation speed of 4-5 times faster. This is because in the latter one, there is a smoothing step. For one case, the NLEO method has clearly a lower accuracy (68.6\%). The EEG of this patient has higher activity periods which are not seen by clinicians as bursts. Because of a smoothing step, this higher activity is smoothed out. Afterwards, detection is performed with fixed amplitude in contrast to the proposed patient dependent amplitude (meth1).

The mean accuracy for meth2 (based on envelope detection) is $78.8 \%$. False positives are introduced by movement artefacts as there is only an amplitude threshold on the envelope values (around $30 \mu \mathrm{V})$. These are bursts detected by the algorithm, but not by the expert. Besides that, a training phase is needed for this algorithm to tune the different parameter values for the algorithm settings, which is not optimal here because the dataset is limited. This method has around the same computation time as the one based on NLEO. Hence, it can be concluded that the developed algorithm is accurate, robust and fast.

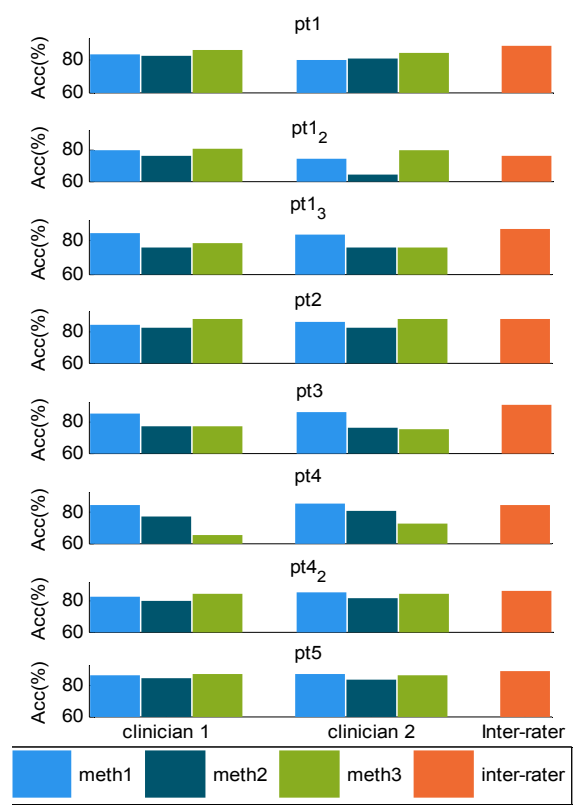

Figure 3: Accuracy obtained by comparing sample by sample clinical labelling with automatic detections of bursts. In the first column labelling of clinical expert 1 are compared with the labelling of the three methods, whereas in the second column this is done for clinical expert 2. In the third column labelling of both clinicians are compared. This analysis is done for 5 patients, where $\mathrm{pt} 1_{2}$ stands for the second measurement of patient 1 at day 14 and pt $1_{3}$ for the measurement when the patient could leave the hospital.

\subsection{Comparison of Features}

Figure 4 summarizes the calculated features for the detection of bursts and IBIs by three previously 

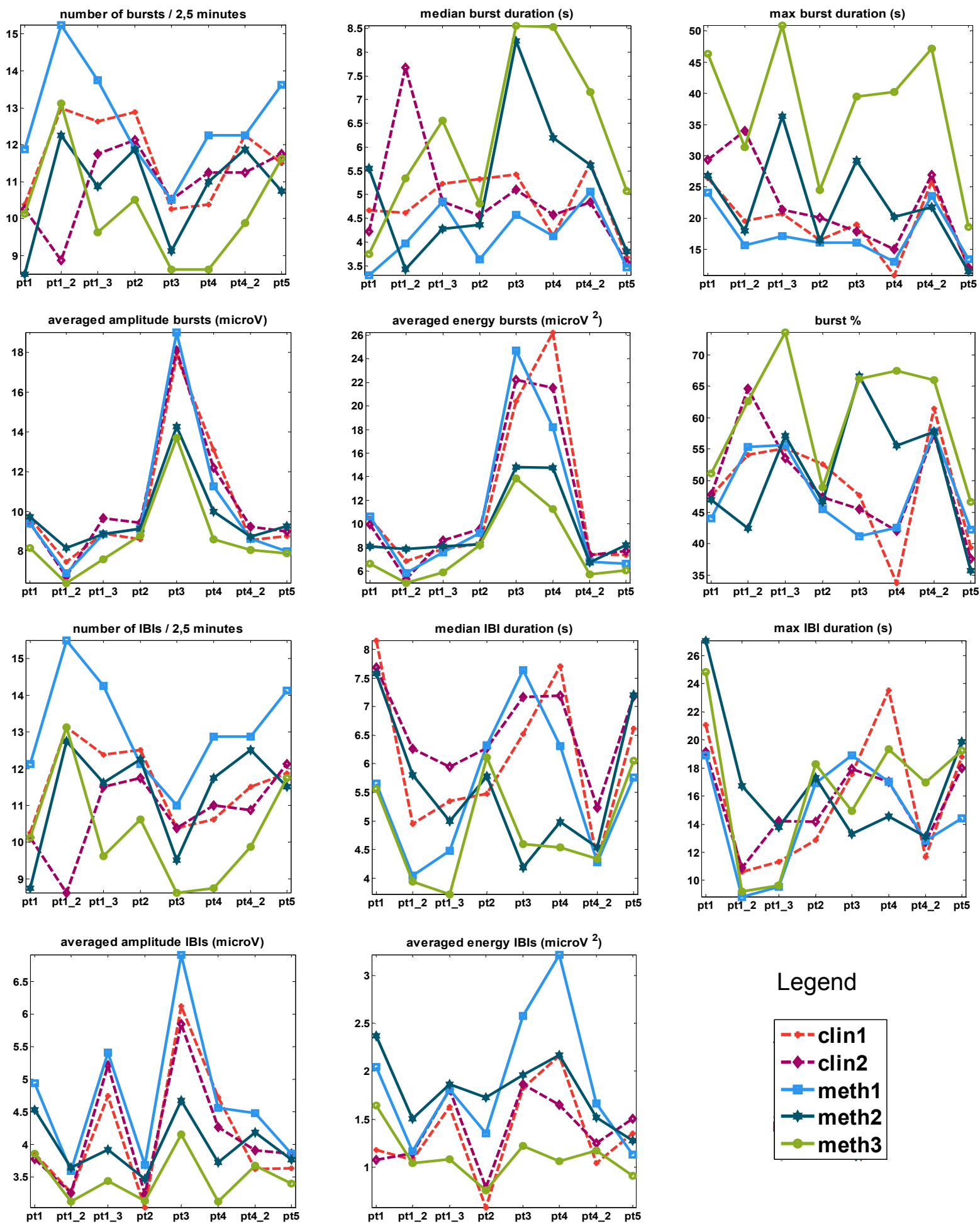

Legend

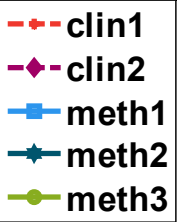

Figure 4: Results for each feature after clinical labelling of bursts and applying different burst detection methods (meth1: based on line length calculation, meth2: based on envelope calculation, meth3: using Non-Linear Energy Operator). 
described methods and two clinicians. Mean duration is not shown because it has similar values as the median duration, where the median is more robust. Detection is subjective from rater to rater, what always leads to a difference between the values between the raters. In other words, there is no golden standard for the description of bursts and IBIs.

Nevertheless, a clear distinction between bursts and IBIs can be found by looking at two features: average amplitude and average energy operator. Namely, the average amplitude for IBIs is smaller than the average amplitude for bursts. This is always the case, for all methods and for all raters. This is also true for values of the average energy operator. IBIs contain less energy than bursts.

Although burst $\%$ was considered as the statistically most significant parameter for correlations between all raters (Palmu et al., 2010), representation of this feature in Figure 4 shows that the correlation between values obtained by the NLEO algorithm and the clinicians is not that high. Especially the line length method gives a high correlation.

Maximum IBI duration has been reported to decrease as the postmenstrual age increases (Hayakawa et al., 2001). In the present study, the maximum IBI decreases from the first measurement to the second measurement two weeks later for patients 1 and 4 (Figure 4). Not only a decrease of the maximum IBI duration, but also a decrease of the median IBI value has a good prognostic value for good neurological outcome. Values for this parameter decrease as well for patients 1 and 4 .

By checking differences between values obtained for clinical and algorithm detection, it can be said that the developed algorithm approximates well the values of the parameters after the clinical detection.

\section{CONCLUSIONS}

The developed algorithm is a successful strategy to detect patterns in the premature EEG, like bursts and the intervals between them. The automated analysis of EEG provides possibilities to look over a longer period of time and over various records at different points in time. Also, assessment of the evolution over time of the unique characteristics of the EEG is very valuable. Consequently, good approximation of clinical features is of high importance. Thereby, it aggregates the experience and trained eyes of more clinical doctors and researchers in an overarching model. Future work will focus on fine-tuning the algorithm based on a larger dataset of validated EEG segments. Additionally, more features and their clinical relevance have to be explored. Such a detection algorithm would dramatically improve the overall assessment in the NICU for EEG diagnosis.

\section{ACKNOWLEDGEMENTS}

Research supported by

Research Council KUL: GOA MaNet, PFV/10/002 (OPTEC), IDO 08/013 Autism, several PhD/postdoc \& fellow grants;

Flemish Government: FWO: $\mathrm{PhD} /$ postdoc grants, projects: G.0427.10N (Integrated EEG-fMRI), G.0108.11 (Compressed Sensing) G.0869.12N (Tumor imaging); IWT: TBM070713-Accelero, TBM070706-IOTA3， TBM080658-MRI (EEGfMRI), TBM110697-NeoGuard, PhD Grants; IBBT; MDV is supported by an Alexander von Humboldt stipend.

Belgian Federal Science Policy Office: IUAP P7/ (DYSCO, 'Dynamical systems, control and optimization', 2012-2017); ESA AO-PGPF01, PRODEX (CardioControl) C4000103224.

EU: RECAP 209G within INTERREG IVB NWE programme, EU HIP Trial FP7-HEALTH/ 2007$2013\left(n^{\circ} 260777\right)$.

\section{REFERENCES}

Accardo, A., Affinito, M., Carrozzi, M., et al., 1996. Use of the fractal dimension for the analysis of electroencephalographic time series. In Biological Cybernetics, 77: p. 339-350.

De Vos, M., Deburchgraeve, W., Cherian, P.J., et al., 2011. Automated artifact removal as preprocessing refines neonatal seizure detection. Clinical Neurophysiolog, 122, p. 2345-2354.

Esteller, R., Echauz, J., Tcheng, T., et al., 2001. Line length: An efficient feature for seizure onset detection. Papers from $23^{\text {rd }}$ annual International Conference of the IEEE Engineering in Medicine and biology Society, Istanbul, Turkey.

Hayakawa, M., Okumura, A., Hayakawa, F., et al., 2001. Background electroencephalographic (EEG) activities of very preterm infants born at less than 27 weeks gestation: a study on the degree of continuity. In Archives of Disease in Childhood - Fetal and Neonatal Edition, 84: p. 163-167.

Jennekens, W., Ruijs, L. S., Lommen, C. M. L., et al., 2011. Automatic burst detection for the EEG of the preterm infant. In Physiological Measurement, 32: p. 1623-1637.

Le Bihannic, A., Beauvais, K., Busnel, A., et al., 2011. Prognostic value of EEG in very premature newborns. 
In Archives of Disease in Childhood - Fetal and Neonatal Edition, 97 no.2: p. 106-109.

Palmu, K., Stevenson, N., Wikström, S., et al., 2010. Optimization of an NLEO-based algorithm for automated detection of spontaneous activity transients in early preterm EEG. In Physiological Measurement, 31: p. 85-93.

Palmu, K., Wikström, S., Hippeläinen, E., et al., 2010. Detection of 'EEG bursts' in the early preterm EEG: Visual vs. Automated detection. In Clinical Neurophysiology, 121: p. 1015-1022.

Särkelä, M., Mustola, M., Seppänen, T., et al., 2002. Automatic analysis and monitoring of burst suppression in anesthesia. In Journal of Clinical Monitoring and computing, 17: p. 125-134.

Vanhatalo S., Kaila, K., 2006. Development of neonatal EEG activity: from phenomenology to physiology. In Seminars in fetal \& neonatal medicine, 11 no. 6: $\mathrm{p}$. 471-478. 